Int. J. Dev. Biol. 56: 711-718 (2012)

doi: $10.1387 / \mathrm{ijdb} .113390 \mathrm{~mm}$

\title{
Loss of plakophilin 2 disrupts heart development in zebrafish
}

\author{
MIRIAM A. MORIARTY ${ }^{1, \#,}$ REBECCA RYAN ${ }^{2, \#,}$, PIERCE LALOR ${ }^{3}$, \\ PETER DOCKERY ${ }^{3}$, LUCY BYRNES ${ }^{2}$ and MAURA GREALY*,1 \\ ${ }^{1}$ Pharmacology and Therapeutics, School of Medicine, ${ }^{2}$ Biochemistry, School of Natural Sciences and \\ ${ }^{3}$ Anatomy, School of Medicine, National University of Ireland, Galway
}

\begin{abstract}
The desmosomal armadillo protein plakophilin 2 is the only plakophilin expressed in the heart, and mutations in the human plakophilin $\mathbf{2}$ gene result in arrhythmogenic right ventricular cardiomyopathy. To investigate loss of function, we knocked down plakophilin 2 by morpholino microinjection in zebrafish. This resulted in decreased heart rate, cardiac oedema, blood pooling, a failure of the heart to pattern correctly and a twisted tail. Co-injection of plakophilin 2 mRNA rescued the morphant phenotype, indicating the specificity of the knockdown. Desmosome numbers were decreased in morphant hearts and the plaque and midline structures of the desmosomes in the intercalated discs were disrupted when examined by electron microscopy. cm/c2 and vmhcexpression at 48 hours post-fertilization (hpf) showed incomplete looping of the heart in morphant embryos by whole mount in situ hybridization, and bmp4 expression was expanded into the ventricle. The domain of expression of the heart marker $n k x 2.5$ at 24 hpf was expanded. At the 18 somite stage, expression of the cardiogenic gene lefty 2 was abolished in the left cardiac field, with concomitant increases in bmp4, spaw and lefty1 expression, likely resulting in the looping defects. These results indicate that plakophilin 2 has both structural and signalling roles in zebrafish heart development.
\end{abstract}

KEY WORDS: plakophilin 2, adhesion, zebrafish, cardiac laterality

\section{Introduction}

Plakophilins are armadillo repeat proteins, which serve as major outer dense plaque proteins in desmosomes; cell-cell junctions which function in maintaining tissue integrity, in signal transduction pathways, and in epidermal organisation. However, the primary role of desmosomes is to resist mechanical stress in tissues such as the skin and heart (Kottke et al., 2006 for review). Plakophilins link the C-termini of desmosomal cadherins to desmoplakin which in turn binds the intermediate filaments of the cytoskeleton (AlAmoudi et al., 2011 for review). Plakophilin 2 is the only plakophilin found in the heart, underlying its importance in maintaining cardiac structural integrity. In cell culture experiments plakophilin 2 has been shown to have varied roles: it can enter the nucleus where it interacts with the RNA polymerase III holoenzyme, suggesting a role in translation; it can also associate with beta-catenin and increase Wnt signalling in a Topflash luciferase reporter assay in colon carcinoma cells, suggesting that it might interact with the Wnt signalling pathway (Mertens et al., 1996, 2001; Chen et al., 2002); and it can act as a scaffold for protein kinase C-alpha signalling (Bass-Zubek et al., 2008). Loss of plakophilin 2 leads to decreased sodium current and sodium channel remodelling in cultured cardiac myocytes (Sato etal., 2009, 2011), with an increase in re-entrant activity (Deo et al., 2011), and increased expression of desmocollins 2 and 3 , cell proliferation and lipid accumulation (Matthes et al., 2011).

In humans plakophilin 2 mutations are linked to arrhythmogenic right ventricular cardiomyopathy (ARVC), a cardiac condition characterised by cardiac arrhythmias, fibrofatty replacement of cardiomyocytes and sudden death (reviewed by Herren et al., 2009). To date, 224 pathogenic plakophilin 2 variants have been

\footnotetext{
Abbreviations used in this paper: ARVC, arrhythmogenic right ventricular cardiomyopathy; bmp4, bone morphogenic protein 4; bpm, beats per minute; bp, base pairs; cmlc2, cardiac myosin light chain 2; EGFP, enhanced green fluorescent protein; flil, Friend leukemia integration 1 transcription factor; hpf, hours post fertilization; oep, one-eyed pinhead; PKP2; plakophilin 2; RTPCR, reverse transcriptase polymerase chain reaction; spaw, southpaw; vmhc, ventricular myosin heavy chain.
}

\footnotetext{
*Address correspondence to: Maura Grealy. Pharmacology and Therapeutics, National University of Ireland Galway, University Road, Galway, Ireland. Tel: +353-91-493-012. Fax: +353-91-495.586. E-mail: maura.grealy@ nuigalway.ie

"Co-authorship: Miriam Moriarty and Rebecca Ryan contributed equally to this work.
}

Supplementary Material (1 table and 4 videos) for this paper is available at: http://dx.doi.org/10.1387/ijdb.113390mm 
reported (van der Zwaag et al., 2009; ARVD/C genetic variants database http://www.arvcdatabase.info). ARVC hearts have fewer desmosomes and gap junctions in the intercalated discs (Basso et al., 2006; Asimaki et al., 2007). Asimaki et al., (2009) have shown that junctional plakoglobin is reduced inARVC hearts including those with mutated plakophilin 2. The replacement of cardiac myocytes by fibrous and fatty tissue is also characteristic of ARVC. This likely involves differentiation of cardiac progenitor cells to adipocytes, a process that can be caused by suppression of canonical Wnt signalling (Lombardi et al., 2009). We and others have shown that plakoglobin, another armadillo protein, is inhibitory to Wnt signalling and its knockdown reduces the number of desmosomes in the intercalated discs (Martin et al., 2009; Lombardi et al., 2009).

Loss-of-function animal models have been used to unravel the mechanisms by which mutations in plakophilin 2 and other desmosomal mutations cause ARVC. The depletion of plakophilin 2 in the cardiac muscle of mice results in defective heart morphogenesis followed by cardiac rupture and blood leakage, and defects in the cellular junctions with embryonic lethality at midgestation (Grossmann et al., 2004).

Due to this embryonic lethality in mice, we chose the zebrafish model system to examine the role of plakophilin 2 in cardiovascular development because zebrafish embryos can survive for a few days without a functioning cardiovascular system (Stainier, 2001 for review). We have previously identified zebrafish plakophilin 2 and localised its expression to the developing heart (Moriarty et al., 2008). We hypothesised that plakophilin 2 is integral to correct cardiac morphology and adhesion in zebrafish, with possible Wnt signalling effects. Here we report that the loss of plakophilin 2 results in embryos with severe cardiac defects. In morphant embryos the atrium is enlarged and the ventricle fails to loop. The expression of lefty2, a cardiogenic gene important in cardiac laterality and looping, is abolished at the 18-somite stage. Adhesion junctions of the intercalated discs are lower in number and have an increased intercellular space. These results indicate that plakophilin 2 plays an important role in the modulation of cardiogenic gene expression as well as in the structural integrity of the heart in zebrafish.

\section{Results}

\section{Phenotype generated by knocking down plakophilin 2 in zebrafish}

Embryos were injected with $2.5 \mathrm{ng}, 5 \mathrm{ng}$ or $7.5 \mathrm{ng}$ of target morpholino or mismatch control morpholino and the resulting phenotypes were analysed at 24-, 48-, and 72 hours post fertilization (hpf). Morphant embryos were divided into four categories based on their phenotype-normal, mild, intermediate or severely affected (Fig. 1 A-D). Normal embryos resembled their control siblings while mildly affected embryos had slight cardiac oedema. Intermediately affected morphants had increased cardiac oedema, blood pooling on the yolk and abnormal ventricles. Severely affected morphants had all of these with severe cardiac oedema and heartstring. At
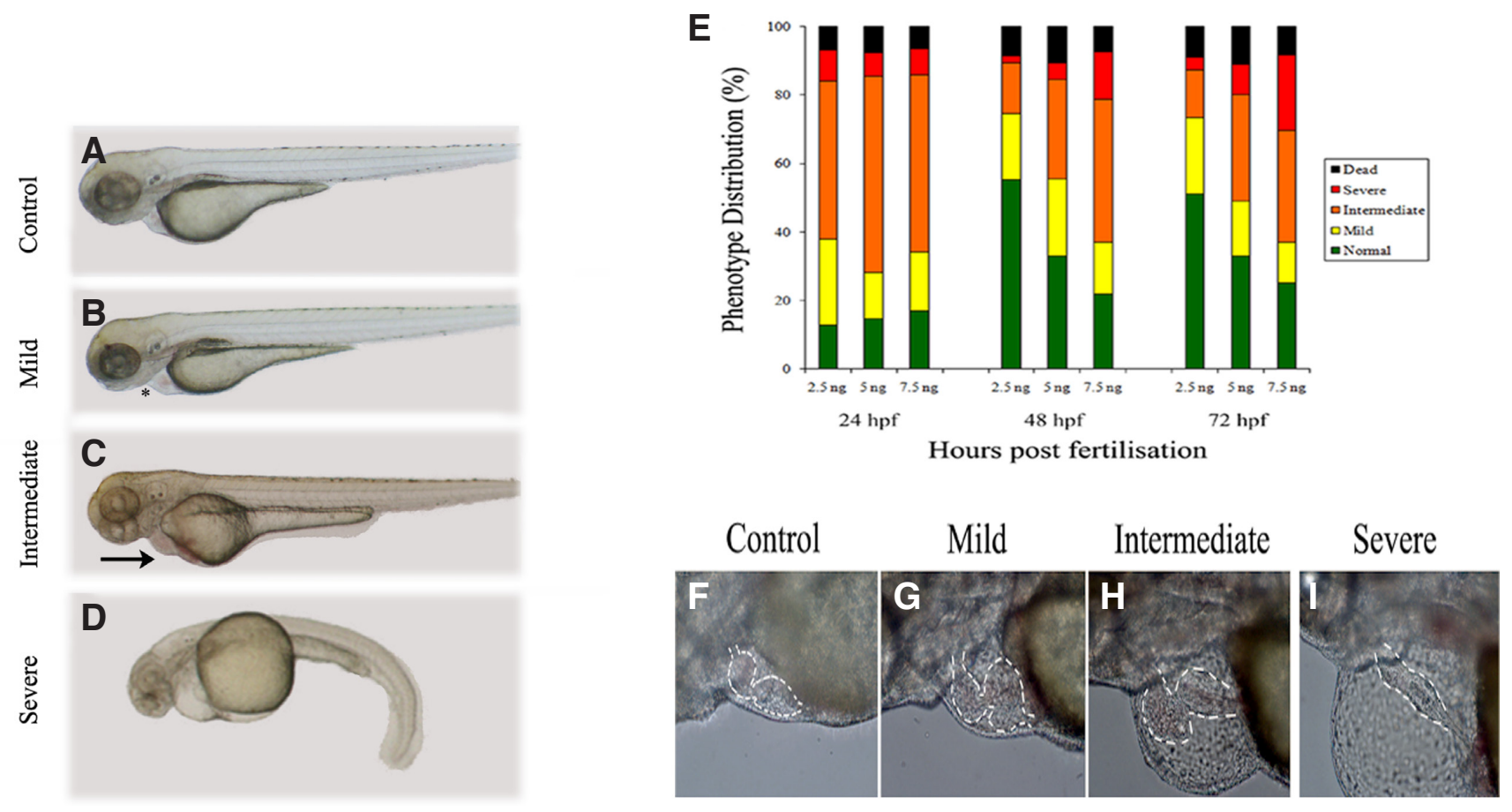

Fig. 1. Effects of plakophilin 2 knockdown on zebrafish development. $5 \mathrm{ng}$ control $5 \mathrm{bp}$ mismatch morpholino (A) or plakophilin 2 morpholino (B,C,D), were injected into embryos at the 1- cell stage. Phenotypes were observed at 72 hpf. (B) Mildly affected morphant embryos displayed pericardial oedema (asterisk) compared to controls. Morphant embryos in the intermediate category (C) exhibited oedema coupled with blood pooling on the yolk (arrow). (D) Severely affected morphants showed increased cardiac oedema, blood pooling and a kinked tail. (E) Either $2.5 \mathrm{ng}, 5 \mathrm{ng}$ or $7.5 \mathrm{ng}$ of plakophilin 2 morpholino were injected into embryos at the 1-cell stage. The resulting morphant phenotypes were scored at 24-, 48- and 72 hpf. The distribution of the four classifications of phenotypes - normal (green), mild (yellow), intermediate (orange), severe (red) and dead (black) - is given in percentages. An average of 60 embryos were injected per experiment, $n=3$ separate experiments. (F-I) Lateral view of 5 ng plakophilin 2 morphant embryo hearts at $72 \mathrm{hpf}$ with anterior to the left and dorsal to the top. (F) Control-injected embryos were normal; morphant phenotypes ranged from (G) mildly affected to (H) intermediate to (I) severely affected. Outline of heart shape is indicated in each phenotype. 
a dose of $2.5 \mathrm{ng}, 24 \mathrm{hpf}$ embryos showed disruption to the tail and yolk extension and at $48 \mathrm{hpf}$ cardiac oedema and a kinked tail. However, at this dose the effects did not persist to 48 and 72 hpf in a large subset of embryos (Fig 1E). Injection of $5 \mathrm{ng}$ of the morpholino caused a kinked tail and disruption to the borders of the anterior neural structures at $24 \mathrm{hpf}$. At $48 \mathrm{hpf}$ the tail was still slightly deformed and there was considerable cardiac oedema with blood pooling on the yolk in a subset of these embryos. At 72 hpf the tail defects were less severe while the cardiac phenotype was more pronounced. The loss of plakophilin 2 in these embryos significantly lowered the heart rate from $138 \pm 3 \mathrm{bpm}$ to $110 \pm 8 \mathrm{bpm}$ at $48 \mathrm{hpf}$ and from $145 \pm 4 \mathrm{bpm}$ to $116 \pm 5$ at $72 \mathrm{hpf}(\mathrm{n}=20$ in each group, $P<0.05)$. Embryos in the $7.5 \mathrm{ng}$ treatment group had similar phenotypes to those treated with $5 \mathrm{ng}$ - anterior neural and tail defects, and cardiac disruption. However, embryos injected with $7.5 \mathrm{ng}$ of the control morpholino also had defects, whereas those injected with $5 \mathrm{ng}$ of the control morpholino appeared normal. Therefore, $5 \mathrm{ng}$ was selected as the optimum dose for subsequent experiments. At this dose a range of cardiac phenotypes occurred, from normal to oedema and a failure to loop (Fig. 1 F-I; Supplementary videos 1-4).

\section{Rescue of the morphant phenotype by co-injection of pla- kophilin 2 RNA}

To confirm that the observed morphant phenotype was specific to the loss of plakophilin 2, embryos were co-injected with histidinetagged zebrafish plakophilin 2 mRNA and either control or target morpholino. A dose response study determined the optimal dose to be $125 \mathrm{pg}$ of plakophilin 2 (Fig 2E). This dose restored the morphant phenotype to that seen in control embryos (Fig. 2 A-D), whereas embryos co-injected with $62.5 \mathrm{pg}$ retained the morphant phenotype, while those receiving $250 \mathrm{pg}$ were severely disrupted or dead. Co-injection of $125 \mathrm{pg}$ of the RNA construct also restored the heart rate of embryos to normal $-140 \pm 1 \mathrm{bpm}$ at $48 \mathrm{hpf}$ and $149 \pm 1$ bpm at $72 \mathrm{hpf}$ ( $\mathrm{n}=10$ in each group, $P<0.05$ vs morphants). Western blot analysis confirmed the translation of the co-injected histidine-tagged zebrafish plakophilin 2. An anti-histidine antibody was used to visualise the plakophilin 2 band at approximately 90 $\mathrm{kDa}$ in lysates from the target and control morphant embryos that were co-injected with the RNA construct, whereas this band was absent in the samples from embryos injected with morpholino alone (Fig. 2F). This rescue of the morphant phenotype confirms that the morpholino-induced defects are specific to the loss of plakophilin 2.

\section{Circulation and angiogenesis in plakophilin 2 morphants}

As morphant embryos exhibited blood pooling on the yolk, we examined blood circulation and angiogenesis in the developing embryos using $\mathrm{O}$-dianisidine, an indicator of the peroxidase activity of haemoglobin. Live plakophilin 2 morphant embryos and sibling controls were stained at $72 \mathrm{hpf}$, allowing for the visualisation of the circulating blood. In control-injected embryos blood was present in the anterior blood vessels, the heart, the common cardinal vein, the dorsal aorta and posterior cardinal vein (Fig. 3A). Although blood pooling was observed on the yolk and around the heart of morphant embryos, circulation was unaffected (Fig. 3B). We then examined the vasculature in control and morphant embryos at $48 \mathrm{hpf}$ and $72 \mathrm{hpf}$ to assess angiogenesis using the transgenic fli1:EGFP fish (Lawson and Weinstein, 2002). Blood vessel number and pattern were similar in control and morphant embryos indicating that
COMO
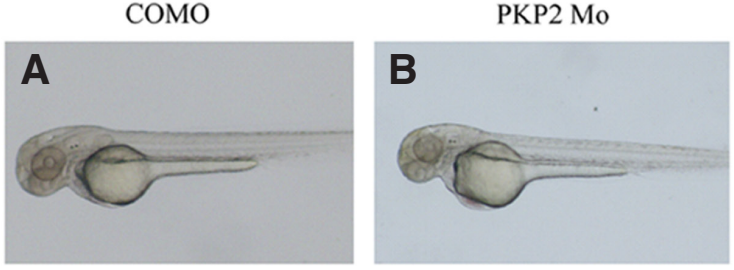

COMO \& 125 pg RNA

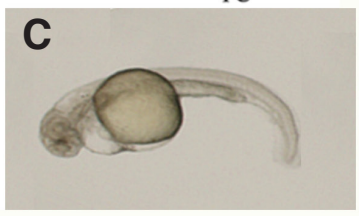

PKP2 Mo \& 125 pg RNA
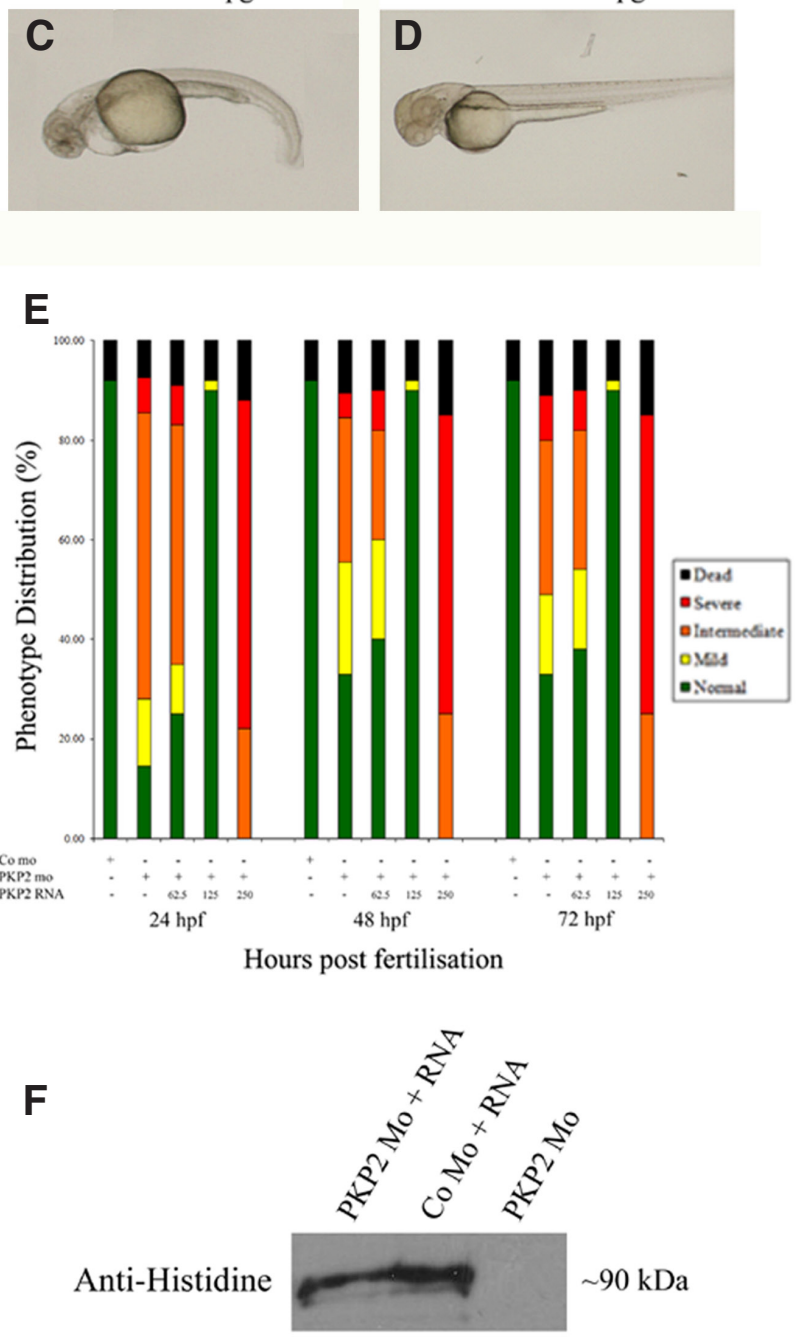

Anti-Alpha tubulin $-\sim 50 \mathrm{kDa}$

Fig. 2. Phenotype of embryos co-injected with plakophilin 2 RNA. $(\mathbf{A}, \mathbf{B})$ Confirmation of the control and morphant phenotypes seen in the initial knockdown study. Co- injection of $125 \mathrm{pg}$ of plakophilin 2 RNA with the control morpholino led to head, cardiac and trunk disruption (C), while co-injection with the target morpholino rescued the morphant phenotype (D) at 72 hpf. (E) Phenotype distribution of embryos injected with $5 \mathrm{ng}$ of control or plakophilin 2 morpholino alone or along with 62.5 pg, 125 pg or $250 \mathrm{pg}$ or plakophilin 2 RNA. The affected embryos were classified as normal, mild, intermediate, severe or dead. (F) Western blot analysis confirmed the translation of histidine-tagged plakophilin 2 in 24 hpf embryo lysates with an anti-histidine antibody at the expected size of $90 \mathrm{kDa}$. Alpha tublin was used as a loading control. 

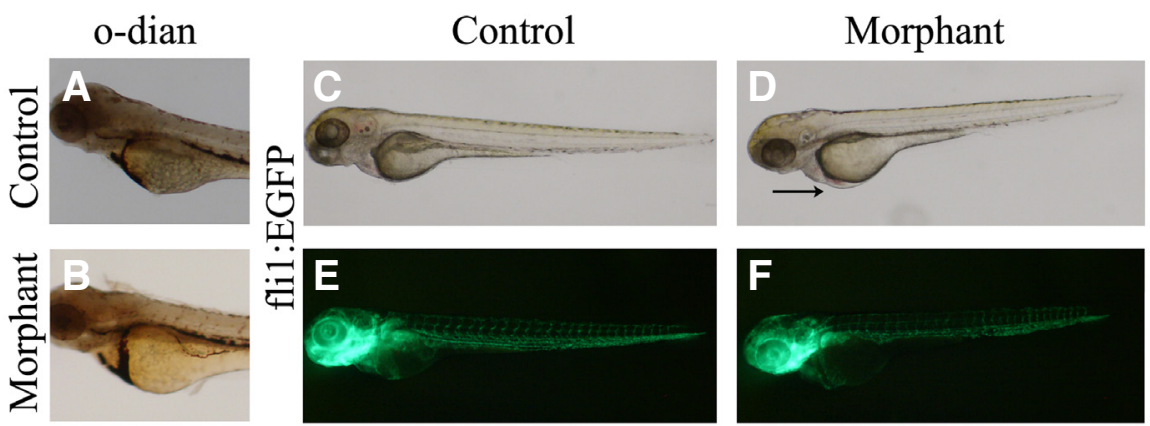

Fig. 3 (Left). Blood pooling in morphant embryos. $(\mathbf{A}, \mathbf{B})$ Lateral view of O-dianisidine stained $72 \mathrm{hpf}(\mathbf{A})$ control and (B) morpholino injected embryos. Blood was present in the anteriorblood vessels, the heart, the common cardinal vein, the dorsal aorta, and posterior cardinal vein. In the morphant embryos, blood was pooled on the yolk prior to entering the heart. (C-F) The endothelial specific transgenic fli1:EGFP embryos injected with (E) control or (F) plakophilin 2 morpholino were analysed under light ( $C$ and $D$ ) and fluorescence microscopy ( $E$ and F) for vascular defects at 72 hpf. There was no alteration in vasculature in morphant embryos despite evident blood pooling (arrow).

blood pooling was not due to defects in vasculature and may be a secondary effect of the morphological abnormalities and reduced heart rate seen in our morphants (Fig. 3 C-F).

\section{Cardiac cell junctions in plakophilin 2 morphants}

To fully understand the effects of loss of plakophilin 2 on adhesion in the developing embryo desmosomal structure was investigated using transmission electron microscopy. Plakophilin 2 morphants exhibited specific cardiac defects and so adhesion junctions in the developing zebrafish heart were examined at $72 \mathrm{hpf}$, when the heart is fully formed. In control embryos desmosomes, with their

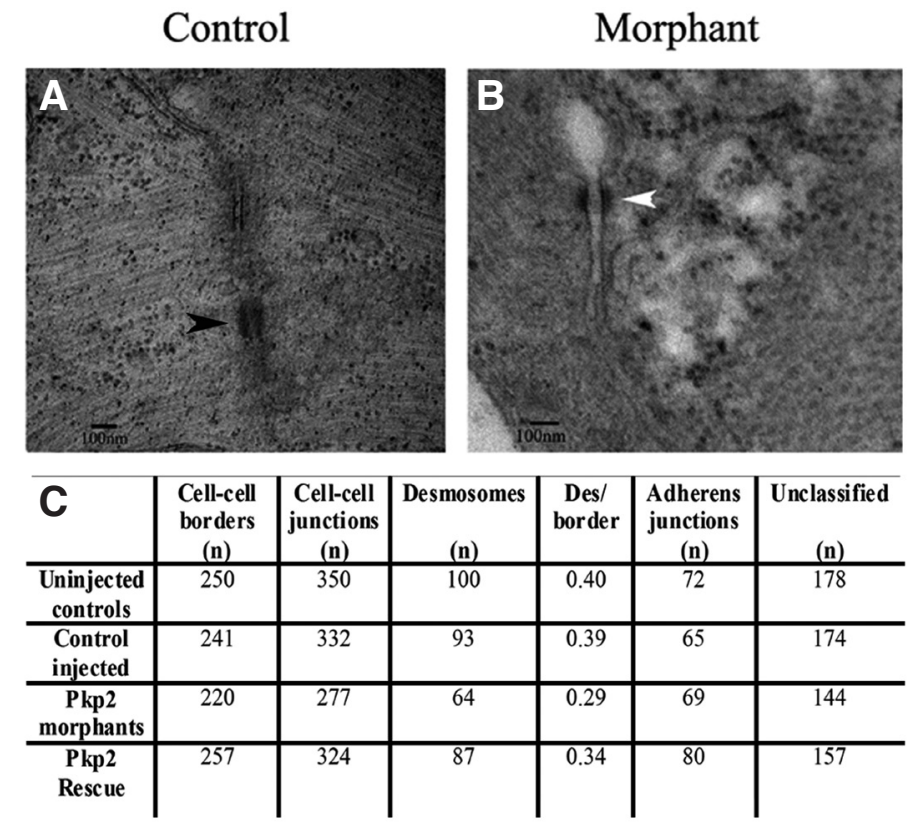

distinctive dense tripartite composition, were easily distinguished from adherens junctions, and were often observed in series at the cell borders (Fig. 4A). In plakophilin 2 deficient embryos the plaque structure was intact at each cell border but the intercellular elements were diminished and the intercellular gap was wider (Fig. 4B). Disruption of the structure of adhesion junctions in morphant embryos was accompanied by a decrease in the number of desmosomes per cell-cell border $\left(P<0.05, \chi^{2}\right.$ test). Adherens junctions were unaffected. Rescuing the plakophilin 2 phenotype

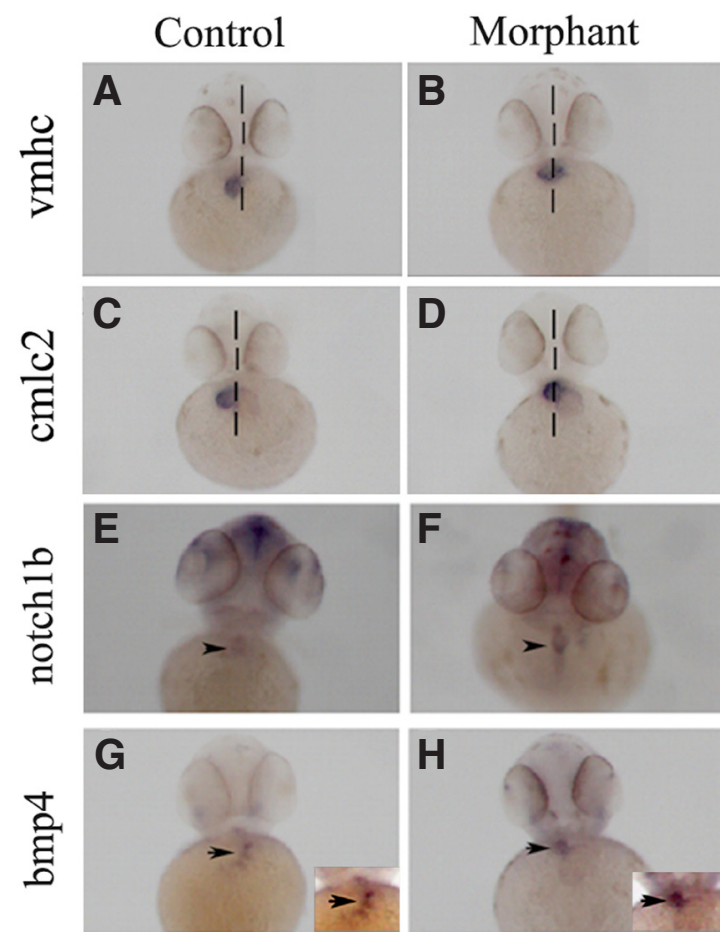

Fig. 4 (Left). The ultrastructure of adhesion junctions in plakophilin 2 morpholino injected embryo hearts. Electron microscopy of the intercalated discs of zebrafish embryo hearts at $72 \mathrm{hpf}$. (A) In control morpholino injected embryos, intercellular junction elements (black arrowhead) were clearly evident. (B) In plakophilin 2 morphant embryos, junctions lacked these intercellular elements (white arrowhead) and the gap between cells was larger. (C) The table details the cell-cell border numbers, the number of adhesion junctions, and the number of each type of junction in control, morphant and rescued embryos. The number of desmosomes per border was decreased in plakophilin 2 morphants compared to controls, with an increase towards normal levels in the rescued embryos. Unclassified indicates adhesion junctions that could not be definitively classed as adherens junctions or desmosomes. $\mathrm{N}=3$ embryos per sample. Scale bar is $100 \mathrm{~nm}$.

Fig. 5 (Right). Expression of cardiac morphology marker genes in plakophilin 2 morphant and control morpholino injected embryos. Ventral views of 48 hpf wholemount embryos with (A-D) the midline denoted by a broken line. (A and B) vmhc delineates ventricle specification at 48 hpf in (A) control and (B) morphant embryos. In morphants the ventricle remained at the midline. (C,D) $\mathrm{cmlc2}$ was expressed in both atrium and ventricle at $48 \mathrm{hpf}$ in (C) control and (D) morphant embryos with midline placement in the latter. (E,G) Expression of both notch $1 \mathrm{~b}$ and bmp4 was restricted to the atrio-ventricular boundary (black arrowheads) in control morpholino injected embryo hearts; (F and $H$ ) in morphant embryos, both cardiac valve markers were expanded towards the ventricle (black arrows). The numbers of embryos with the displayed phenotype were (A) 67/70 (B) 34/61 (C) 68/70 (D) 28/45 (E) 47/47 (F) 28/49 (G) 49/49 (H) 43/67, numbers of morphant embryos showing the phenotype reflect the proportion of affected embryos (Fig. 1). 
A

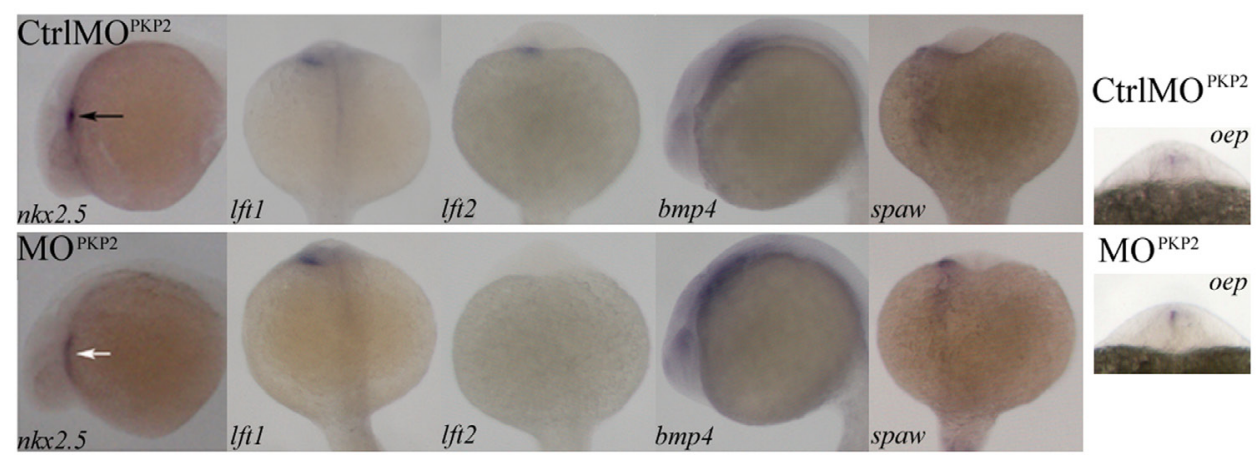

B

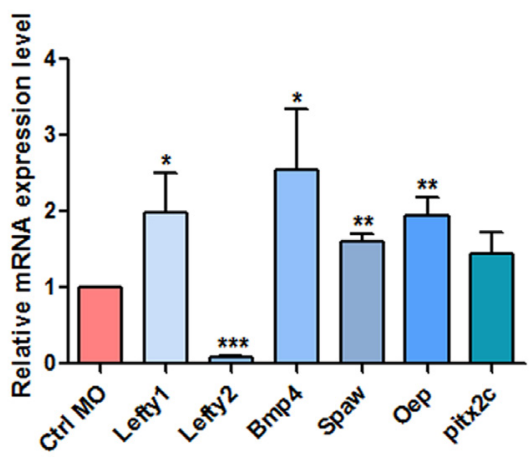

Fig. 6. Expression of the cardiogenic marker genes in plakophilin 2 morphant and control morpholino injected embryos. (A) Wholemount in situ hybridization lateral view of nkx2.5 at $24 \mathrm{hpf}$ and bmp4 at 18 somites. Dorsal views of lefty 1 (Ift1), lefty 2 (Ift2), spaw and oep at 18 somites. nkx2.5 was expressed to the left of the midline in control injected embryos - black arrow; nkx2.5 signal intensity was reduced in morphant embryos, but in the correct location with an expanded domain - white arrow. Expression of lefty 1 was unaffected in morpholino injected embryos while the expression domain of oep was expanded and the intensity of bmp4 and spaw was increased. lefty 2 expression was absent in plakophilin 2 morphant embryos compared to controls. The numbers of embryos with the displayed phenotype were: nkx2.5 control 29/29, nkx2.5 morphant 26/39; Ift1 control 44/54, Ift1 morphant 47/63; Ift2 control 28/34, Ift2 morphant 29/35; bmp4 control 34/36, bmp4 morphant 25/32; spaw control 50/57, spaw morphant 39/47; oep control 42/48, oep morphant 36/40. (B) qRTPCR expression of genes involved in cardiac laterality at 18 somites. lefty 1 , bmp4, spaw, oep were significantly upregulated and lefty2 expression was significantly decreased in morphant embryos compared to control injected embryos ${ }^{*} \mathrm{P}<0.05$, ${ }^{*} *$ $\mathrm{P}<0.005, * * * \mathrm{P}<0.0001)$.

increased the numbers of desmosomes in the heart compared to morphant embryos (Fig. 4C).

Desmosomes are important in the skin as well as the heart and are normally distributed in a 'zipper' pattern along the cell-cell border. In contrast to the effect seen in the heart, desmosomes in the skin of morphant embryos remained intact (data not shown), likely reflecting the ability of plakophilins 1 or 3 to compensate for loss of plakophilin 2 in the skin but not in the heart, where only plakophilin 2 is expressed.

\section{Role of plakophilin 2 in cardiac looping and chamber devel- opment}

The chamber-specific markers, cardiac myosin light chain two (cmlc2) and ventricular myosin heavy chain (vmhc), were used to visualise chamber formation and heart looping at $48 \mathrm{hpf} . \mathrm{cm} / \mathrm{c} 2$ marks both atrium and ventricle while $v m h c$ is confined to the ventricle (Yelon et al., 1999). The expression patterns of both these markers showed incomplete looping of the morphant hearts, with the ventricle placed along the midline above the atrium instead of being placed on the right hand side (Fig. 5 A-D). cmlc2 expression also showed that the developing atrium appeared larger in the plakophilin 2 morphants (Fig. 5D). Correct formation of cardiac valves relies on the restriction of the endocardial marker notch $1 \mathrm{~b}$ and the myocardial marker bone morphogenic protein 4 ( $b m p 4)$ to the future cardiac valve regions by $48 \mathrm{hpf}$ and $37 \mathrm{hpf}$ respectively (Westin and Lardelli, 1997; Walsh and Stainier, 2001). In plakophilin 2 morphants notch $1 b$ expression was markedly expanded in the ventricle. bmp4 was expanded into the ventricle (Fig. $5 \mathrm{E}-\mathrm{H}$ ). However, the valves appeared normal when the structure of 72 hpf control and morphant hearts was examined using electron microscopy (data not shown).

\section{Role of plakophilin 2 in cardiogenic gene expression}

We used several cardiac marker genes to examine heart morphogenesis in our morphant embryos by whole-mount in situ hybridization and by quantitative RTPCR. $n k x 2.5$, one of the earliest cardiac precursor markers, was used to examine cardiac precursor migration and heart tube formation (Chen and Fishman, 1996). It is normally positioned in a discrete area on the left of midline in the anterior of the embryo at $24 \mathrm{hpf}$ (Fig. 6A, black arrow). In plakophilin 2 morphants it was expressed on the correct side of the midline but the intensity of the signal was reduced with an expanded expression domain (Fig. 6A, white arrow). This led us to examine the expression of other cardiogenic genes at the 18-somite stage. The most striking effect was the absence of lefty2 from the left cardiac field in morphant embryos. Other genes in the Nodal cascade, bmp4, southpaw (spaw) and lefty1 were expressed in the correct location but intensity of expression was increased. This was confirmed by qRTPCR, which showed increased expression of bmp4, spaw, lefty1 and one-eyed pinhead (oep), whereas lefty2 expression was practically abolished (Fig. 6B). This reflects the requirement of bmp4 for left-sided expression of spaw, which in turn induces left-sided expression of bmp4 and lefty 1 , whereas lefty 1 and lefty2 negatively regulate each other (Bisgrove et al., 1999; Chocron et al., 2007; Bakkers et al., 2009). To examine if these effects are early or late events in cardiogenesis, we examined bmp4 expression at sphere, shield and $48 \mathrm{hpf}$ in addition to the 18-somite stage by qRTPCR. The only significant change in expression was at 18-somites indicating that this effect occurs late in cardiogenesis.

\section{Discussion}

This study has shown that plakophilin 2 is essential for cardiovascular development. Knockdown of plakophilin 2 resulted in cardiac oedema, blood pooling, failure of the heart to loop, a decrease in heart rate and abnormal desmosomes in the heart. In addition, we report for the first time that plakophilin 2 affects cardiac laterality. Thus knockdown abolished lefty2 expression, with concomitant increases in expression of Nodal pathway genes, which may be the mechanism by which cardiac looping is affected.

The cardiac defects that we saw following loss of plakophilin 
2, including cardiac oedema, blood pooling and decreased heart rate are similar to those found following loss of other desmosomal proteins in zebrafish. Knockdown of desmocollin 2 led to decreased heart rate, cardiac oedema and a loss of desmosomes (Heuser et al.,2006); similarly a loss of plakoglobin resulted in cardiac oedema, decreased heart rate and chamber size, and reflux of blood between chambers (Martin et al., 2009). When plakophilin 2 is ablated in mice the developing heart morphology is also disrupted, with blood pooling and pericardial oedema (Grossmann et al., 2004).

Injection of wildtype mRNA has been shown to rescue cardiac phenotypes in several studies (Heuser et al., 2006; Martin et al., 2009; Hartnett et al., 2010). The ability of the plakophilin 2 mRNA to rescue the phenotype in a dose-dependent manner in our study shows the specificity of the morpholino knockdown.

Despite the blood pooling on the yolk of plakophilin 2 morphants, the circulation and the developing vasculature were normal. The pooling is more likely a secondary effect due to altered heart morphology.

The decreased numbers and altered morphology of desmosomes seen in plakophilin 2 depleted embryos are in general agreement with studies where desmosomal proteins were ablated (Bierkamp et al., 1999; Heuser et al.,.2006; Hall et al., 2009; Martin et al., 2009).

The cardiac defects may be due to abnormal expression levels of cardiogenic genes. The expression pattern of $n k x 2.5$ in plakophilin 2 morphants was similar to that seen in plakoglobin morphants, with a reduced intensity of expression but normal migration to the midline (Martin et al., 2009). However, in plakophilin 2 morphants the domain of expression was expanded, which may cause the increased size of the atrium seen later in development. $\mathrm{cm} / \mathrm{c} 2$ and $v m h c$ expression showed that the developing ventricle had failed to loop correctly in morphants. Cardiac bmp4 expression was expanded into the ventricle at $48 \mathrm{hpf}$ in plakophilin 2 morphants, similar to the pattern seen in apc mutant embryos which also failed to loop (Lin and Xu 2009; Verhoeven et al., 2011). Early expression of bmp4 was normal; however at the 18-somite stage it was increased, likely controlling the increased expression of spaw and lefty 1 ; this in turn would account for the absence of lefty 2expression in the left cardiac field at 18 somites (Bisgrove et al., 1999; Meno et al., 2001). This indicates that plakophilin 2 has both structural and signalling roles in zebrafish heart development.

\section{Materials and Methods}

\section{Zebrafish maintenance}

Zebrafish, AB strain and Tg(fli1:EGFP; Lawson and Weinstein, 2002) were obtained from the Zebrafish International Resource Centre and maintained under standard conditions at $28^{\circ} \mathrm{C}$ (Westerfield, 2000).

\section{Plakophilin 2 knockdown}

A morpholino against the 5'UTR region of plakophilin 2 was designed by and purchased from GeneTools, Oregon (5'-GTCACCTCTCCAAAGATCGTGTTTC $-3^{\prime}$ ). A five-bp mismatch control morpholino was also used (5'- GTgACgTCTgCAAAcATCGTCTTTC -3'). Morpholinos were resuspended in nuclease free water at a stock concentration of $1 \mathrm{mM}$, and were injected at the 1- to 2-cell stage in a volume of $1 \mathrm{nl}$. Plakophilin 2 and control morpholinos were injected at 2.5-, 5- and $7.5 \mathrm{ng}$. The vehicle control was $1 \mathrm{nl}$ phenol red.

\section{Preparation of mRNA for rescue experiments}

Full length open reading frames (ORF) of plakophilin 2 were amplified using primers that included a histidine tag at the $5^{\prime}$ end of the construct
(ORF-forward primer 5'- AGGTGACGCGGATCCATGCATCATCATCATCATCATCTTAAACCACAC-3' histidine tag underlined; ORF-reverse primer 5'-CCATCTAGATTATTCTCGAGCAGA -3'). The construct was subcloned into pCS2+vector. Capped mRNA was synthesized using mMessage mRNA kit (Ambion) according to manufacturer's instructions.

\section{Wholemount in situ hybridization (WISH)}

Antisense probes were synthesized from linearised plasmid using SP6, T7 or T3 polymerase and a digoxigenin-labelling RNA kit (Roche). Embryos were fixed in $4 \%$ paraformaldehyde (PFA), dehydrated and hydrated through a methanol series $(25 \% ; 50 \% ; 75 \%$ and $100 \%$ for five minutes for each solution) and hybridized with probe $(2 \mu \mathrm{l})$ as per Westerfield (2000) and Hauptmann (1999). The bound probe was detected with BM-Purple AP-Substrate (Roche).

\section{O-dianisidine staining}

Dechorionated embryos were incubated in O-dianisidine staining solution (2.45 mM O-dianisidine, $0.01 \mathrm{M}$ sodium acetate, $\mathrm{pH} 4.5,0.65 \% \mathrm{H}_{2} \mathrm{O}_{2}$, and $40 \%$ ethanol) for 15 minutes in the dark at room temperature. The reaction was stopped by washing with PBST three times for five minutes each, followed by fixing in $4 \%$ paraformaldehyde for one hour. Embryos were briefly washed in methanol series for thirty seconds each and mounted in BBA for viewing (Ransom et al., 1996).

\section{Western blotting}

Deyolked and dechorionated embryos were collected as described by Link et al., (2006) with protease inhibitor cocktail (Sigma) in all solutions. Lysates were snap frozen in liquid nitrogen, thawed on ice and $50 \mu \mathrm{l}$ of lysis buffer (1\% Triton-X 100, $10 \mathrm{mM}$ Tris (pH 7.5), $140 \mathrm{mM} \mathrm{NaCl}, 5 \mathrm{mM}$ EDTA, 2 mM EGTA, $1 \mathrm{mM}$ PMSF and 1\% protease inhibitor cocktail) were added per 100 embryos. To aid yolk removal, Freon $(150 \mu \mathrm{l})$ was added to lysates which were vortexed for $30 \mathrm{~s}$ and centrifuged at $4^{\circ} \mathrm{C}$ for $15 \mathrm{~min}$ at $11,337 \times \mathrm{g}$. Protein $(25 \mu \mathrm{g})$ was resolved by $7.5 \%$ reducing SDS-PAGE followed by western blotting. Antibodies used included anti-histidine (1:1000, Sigma), and anti- $\alpha$-tubulin (1:2000, Sigma) with a goat anti-mouse IgG secondary antibody (1:8000, Sigma).

\section{Transmission electron microscopy (TEM)}

Dechorionated embryos at the desired stage were fixed in $2.5 \%$ gluteraldehyde / $2.5 \%$ PFA and post fixed in $0.01 \%$ osmium tetraoxide. The embryos were dehydrated in an ethanol series and embedded in a low viscosity resin (LV Resin - 48g, VH1 hardener - 16g, VH2 hardener - 36g, LV accelerator - 2.5g - Agar Scientific Ltd.). Embryos were sectioned transversely using a Reichert Jung Ultracut instrument and ultrathin sections (70-90 nm) of interest were selected using light microscopy. Ultrathin sections were stained with uranyl acetate and lead citrate and examined under a Hitachi $\mathrm{H} 7000$ electron microscope.

\section{Real-time quantitative PCR}

Embryos were injected with $5 \mathrm{ng}$ of either control or plakophilin 2 morpholino and collected at 18 somites. RNA was extracted and reverse transcribed using random primers. $1 \mu \mathrm{g}$ of total RNA was used for cDNA synthesis. The cDNA stocks were normalised to $0.2 \mu \mathrm{g} / \mu \mathrm{l}$ for use as template in all PCR reactions. All stocks were checked for good quality by $\beta$-actin PCR. The quantitative RTPCR results were quantified by the delta-delta Ct method. All primer pairs were validated by standard curve (Supplementary table 1 qRTPCR primers). Applied Biosystems SYBR-Green chemistry and 7500 Fast machine were used for all experiments. Elongation factor-1 alpha $(E F 1 \alpha)$ was the reference gene used in all experiments. The protocol was adapted from Bookout et al., (2006).

\section{Statistical analyses}

Results were expressed as mean $\pm S D$. The Shapiro-Wilkes test for normality was used. Differences between groups were evaluated by one way ANOVA and Student-Newman-Keuls post-hoc test. Chi-square tests 
were used to compare the distribution pattern of adhesion junctions. qPCR control versus morpholino groups were analysed using Student's $t$-test for each individual gene tested. A $P$ value of less than 0.05 was considered statistically significant.

\section{Acknowledgements and Funding}

The authors wish to thank Dr. D. Yelon for providing cmlc2 and vmhc probes, Dr. J. Yost for bmp4, lefty1 and lefty2, Dr. M. Lardelli for notch1b, Dr. M. Fishman for nkx2.5, Dr. M. Rebagliati for spaw, Dr. A. Schier for oep. We wish to thank Catherine M. Glynn for fish husbandry. This work was funded by the Millennium Fund, Thomas Crawford Hayes Trust Fund, NUI, Galway, Health Research Board of Ireland grants RP/2005/193 and HRA_POR/2011/25, and Science Foundation Ireland 07/RFP/GEN/F468. We are indebted to a PRTLI4 grant to fund the National Biophotonics and Imaging Platform Ireland that supported the TEM in this research.

\section{References}

AL-AMOUDI, A., CASTANO-DIEZ, D., DEVOS, D.P., RUSSELL, R.B., JOHNSON, G.T. and FRANGAKIS, A.S. (2011). The three-dimensional molecular structure of the desmosomal plaque. Proc Natl Acad Sci USA 108: 6480-6485.

ARVD/C Genetic variants database, University Medical Centre, Groningen [online], available: http://www.arvcdatabase.info (accessed 7th September 2012).

ASIMAKI, A., SYRRIS, P., WICHTER, T., MATTHIAS, P., SAFFITZ, J.E. and MCKENNA, W.J. (2007). A novel dominant mutation in plakoglobin causes arrhythmogenic right ventricular cardiomyopathy. Am J Hum Genet 81: 964-973.

ASIMAKI, A., TANDRI, H., HUANG, H., HALUSHKA, M.K., GAUTAM, S., BASSO, C., THIENE, G., TSATSOPOULOU, A., PROTONOTARIOS, N., MCKENNA, W.J., CALKINS, H. and SAFFITZ, J.E. (2009). A new diagnostic test for arrhythmogenic right ventricular cardiomyopathy. N Engl J Med 360: 1075-1084

BAKKERS, J., VERHOEVEN, M.C. and ABDELILAH-SEYFRIED, S. (2009). Shaping the zebrafish heart: from left-right axis specification to epithelial tissue morphogenesis. Dev Biol 330: 213-220.

BASS-ZUBEK, A.E., HOBBS, R.P., AMARGO, E.V., GARCIA, N.J., HSIEH, S.N., CHEN, X., WAHL, J.K., 3RD, DENNING, M.F. and GREEN, K.J. (2008). Plakophilin 2: a critical scaffold for PKC alpha that regulates intercellular junction assembly. J Cell Biol 181: 605-613.

BASSO, C., CZARNOWSKA, E., DELLABARBERA, M., BAUCE, B., BEFFAGNA, G., WLODARSKA, E.K., PILICHOU, K., RAMONDO, A., LORENZON, A., WOZNIEK, O., CORRADO, D., DALIENTO, L., DANIELI, G.A., VALENTE, M., NAVA, A., THIENE, G. and RAMPAZZO, A. (2006). Ultrastructural evidence of intercalated disc remodelling in arrhythmogenic right ventricular cardiomyopathy: an electron microscopy investigation on endomyocardial biopsies. Eur Heart J27: 1847-1854.

BIERKAMP, C., SCHWARZ, H., HUBER, O. and KEMLER, R. (1999). Desmosomal localization of beta-catenin in the skin of plakoglobin null- mutant mice. Development 126: 371-381.

BISGROVE, B.W., ESSNER, J.J. and YOST, H.J. (1999). Regulation of midline development by antagonism of lefty and nodal signaling. Development 126:3253-3262.

BOOKOUT, A.L., CUMMINS, C.L., MANGELSDORF, D.J., PESOLA, J.M. and KRAMER, M.F. (2006). High-throughput real-time quantitative reverse transcription PCR. Curr Protoc Mol Biol Chapter 15: Unit 1518.

CHEN, J.N. and FISHMAN, M.C. (1996). Zebrafish tinman homolog demarcates the heart field and initiates myocardial differentiation. Development 122: 3809-3816.

CHEN, X., BONNE, S., HATZFELD, M., VAN ROY, F. and GREEN, K.J. (2002). Protein binding and functional characterization of plakophilin 2. Evidence for its diverse roles in desmosomes and beta -catenin signaling. J Biol Chem277: 10512-10522.

CHOCRON, S., VERHOEVEN, M.C., RENTZSCH, F., HAMMERSCHMIDT, M. and BAKKERS, J. (2007). Zebrafish Bmp4 regulates left-right asymmetry at two distinct developmental time points. Dev Biol 305: 577-588.

DEO, M., SATO, P.Y., MUSA, H., LIN, X., PANDIT, S.V., DELMAR, M. and BERENFELD, O. (2011). Relative contribution of changes in sodium current versus intercellular coupling on reentry initiation in 2-dimensional preparations of plakophilin-2-deficient cardiac cells. Heart Rhythm 8: 1740-1748.

GROSSMANN, K.S., GRUND, C., HUELSKEN, J., BEHREND, M., ERDMANN, B., FRANKE, W.W. and BIRCHMEIER, W. (2004). Requirement of plakophilin 2 for heart morphogenesis and cardiac junction formation. J Cell Biol 167: 149-160.

HALL, C., LI, S., LI, H., CREASON, V. and WAHL, J.K., 3RD. (2009). Arrhythmogenic right ventricular cardiomyopathy plakophilin-2 mutations disrupt desmosome assembly and stability. Cell Commun Adhes 16: 15-27.

HARTNETT, L., GLYNN, C., NOLAN, C.M., GREALY, M. and BYRNES, L. (2010). Insulin-like growth factor-2 regulates early neural and cardiovascular system development in zebrafish embryos. Int J Dev Biol 54: 573-583.

HAUPTMANN, G. (1999). Two-color detection of mRNA transcript localizations in fish and fly embryos using alkaline phosphatase and beta-galactosidase conjugated antibodies. Dev Genes Evol 209: 317-321.

HERREN, T., GERBER, P.A. and DURU, F. (2009). Arrhythmogenic right ventricular cardiomyopathy/dysplasia: a not so rare "disease of the desmosome" with multiple clinical presentations. Clin Res Cardiol 98: 141-158.

HEUSER, A., PLOVIE, E.R., ELLINOR, P.T., GROSSMANN, K.S., SHIN, J.T., WICHTER, T., BASSON, C.T., LERMAN, B.B., SASSE-KLAASSEN, S., THIERFELDER, L., MACRAE, C.A. and GERULL, B. (2006). Mutant desmocollin-2 causes arrhythmogenic right ventricular cardiomyopathy. Am J Hum Genet 79: 1081-1088.

KOTTKE, M.D., DELVA, E. and KOWALCZYK, A.P. (2006). The desmosome: cell science lessons from human diseases. J Cell Sci 119: 797-806.

LAWSON, N.D. and WEINSTEIN, B.M. (2002). In vivo imaging of embryonic vascular development using transgenic zebrafish. Dev Biol 248: 307-318.

LIN, X. and XU, X. (2009). Distinct functions of Wnt/beta-catenin signaling in KV development and cardiac asymmetry. Development 136: 207-217.

LINK, V., SHEVCHENKO, A. and HEISENBERG, C.P. (2006). Proteomics of early zebrafish embryos. BMC Dev Biol 6: 1.

LOMBARDI, R., DONG, J., RODRIGUEZ, G., BELL, A., LEUNG, T.K., SCHWARTZ, R.J., WILLERSON, J.T., BRUGADA, R. and MARIAN, A.J. (2009). Genetic fate mapping identifies second heart field progenitor cells as a source of adipocytes in arrhythmogenic right ventricular cardiomyopathy. Circ Res 104: 1076-1084.

MARTIN, E.D., MORIARTY, M.A., BYRNES, L. and GREALY, M. (2009). Plakoglobin has both structural and signalling roles in zebrafish development. Dev Bio/327: 83-96.

MATTHES, S.A., TAFFET, S. and DELMAR, M. (2011). Plakophilin-2 and the migration, differentiation and transformation of cells derived from the epicardium of neonatal rat hearts. Cell Commun Adhes 18: 73-84.

MENO, C., TAKEUCHI, J., SAKUMA, R., KOSHIBA-TAKEUCHI, K., OHISHI, S., SAIJOH, Y., MIYAZAKI, J., TEN DIJKE, P., OGURA, T. and HAMADA, H. (2001). Diffusion of nodal signaling activity in the absence of the feedback inhibitor Lefty2. Dev Cell 1: 127-138

MERTENS, C., KUHN, C. and FRANKE, W.W. (1996). Plakophilins 2a and 2b: constitutive proteins of dual location in the karyoplasm and the desmosomal plaque. J Cell Biol 135: 1009-1025.

MERTENS, C., HOFMANN, I., WANG, Z., TEICHMANN, M., SEPEHRI CHONG, S. SCHNOLZER, M. and FRANKE, W.W. (2001). Nuclear particles containing RNA polymerase III complexes associated with the junctional plaque protein plakophilin 2. Proc Natl Acad Sci USA 98: 7795-7800.

MORIARTY, M.A., MARTIN, E.D., BYRNES, L. and GREALY, M. (2008). Molecular cloning and developmental expression of plakophilin 2 in zebrafish. Biochem Biophys Res Commun 367: 124-129.

RANSOM, D.G., HAFFTER, P., ODENTHAL, J., BROWNLIE, A., VOGELSANG, E., KELSH, R.N., BRAND, M., VAN EEDEN, F.J., FURUTANI-SEIKI, M., GRANATO, M., HAMMERSCHMIDT, M., HEISENBERG, C.P., JIANG, Y.J., KANE, D.A., MULLINS, M.C. and NUSSLEIN-VOLHARD, C. (1996). Characterization of zebrafish mutants with defects in embryonic hematopoiesis. Development 123: 311-319.

SATO, P.Y., MUSA, H., COOMBS, W., GUERRERO-SERNA, G., PATINO, G.A., TAFFET, S.M., ISOM, L.L. and DELMAR, M. (2009). Loss of plakophilin-2 expression leads to decreased sodium current and slower conduction velocity in cultured cardiac myocytes. Circ Res 105: 523-526.

SATO, P.Y., COOMBS, W., LIN, X., NEKRASOVA, O., GREEN, K.J., ISOM, L.L., TAFFET, S.M. and DELMAR, M. (2011). Interactions between ankyrin-g, plakophilin-2, and connexin43 at the cardiac intercalated disc. Circ Res 109: 193-201.

STAINIER, D.Y. (2001). Zebrafish genetics and vertebrate heart formation. Nat Rev Genet 2: 39-48.

VANDERZWAAG, P.A., JONGBLOED, J.D., VANDENBERG, M.P., VANDERSMAGT, J.J., JONGBLOED, R., BIKKER, H., HOFSTRA, R.M. and VAN TINTELEN, J.P. (2009). Agenetic variants database for arrhythmogenic right ventricular dysplasia/ cardiomyopathy. Hum Mutat 30: 1278-1283. 
VERHOEVEN, M.C., HAASE, C., CHRISTOFFELS, V.M., WEIDINGER, G. and BAKKERS, J. (2011). Wnt signaling regulates atrioventricular canal formation upstream of BMP and Tbx2. Birth Defects Res A Clin Mol Teratol 91: 435-440.

WALSH, E.C. and STAINIER, D.Y. (2001). UDP-glucose dehydrogenase required for cardiac valve formation in zebrafish. Science 293: 1670-1673.

WESTERFIELD, M. (2000). The zebrafish book. A guide for the laboratory use of zebrafish (Danio rerio). University of Oregon Press, Eugene.

WESTIN, J. and LARDELLI, M. (1997). Three novel Notch genes in zebrafish: implications for vertebrate Notch gene evolution and function. Dev Genes Evol207:51-63.

YELON, D., HORNE, S.A. and STAINIER, D.Y. (1999). Restricted expression of cardiac myosin genes reveals regulated aspects of heart tube assembly in zebrafish. Dev Biol 214: 23-37.

\section{Further Related Reading, published previously in the Int. J. Dev. Biol.}

Cytoskeletal heart-enriched actin-associated protein (CHAP) is expressed in striated and smooth muscle cells in chick and mouse during embryonic and adult stages

Willemijn van Eldik, Abdelaziz Beqqali, Jantine Monshouwer-Kloots, Christine Mummery and Robert Passier

Int. J. Dev. Biol. (2011) 55: 649-655

Insulin-like growth factor-2 regulates early neural and cardiovascular system development in zebrafish embryos

Lori Hartnett, Catherine Glynn, Catherine M. Nolan, Maura Grealy and Lucy Byrnes Int. J. Dev. Biol. (2010) 54: 573-583

Identification of hoxb1b downstream genes: hoxb1b as a regulatory factor controlling transcriptional networks and cell movement during zebrafish gastrulation Willem M.R. van den Akker, Antony J. Durston and Herman P. Spaink Int. J. Dev. Biol. (2010) 54: 55-62

Movement and commitment of primitive streak precardiac cells during cardiogenesis Lopez-Sanchez, C., Garcia-Masa, N., Ganan, C.M. and Garcia-Martinez, V. Int J Dev Biol (2009) 53: 1445-1455

Isolation and expression analysis of foxj1 and foxj1.2 in zebrafish embryos Aamar, E. and Dawid, I.B.

Int J Dev Biol (2008) 52: 985-991

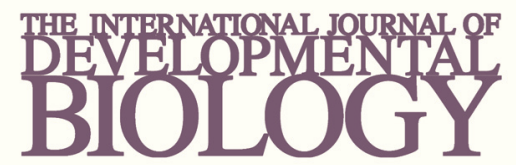

Volume 54 Nos. $6 / 7$
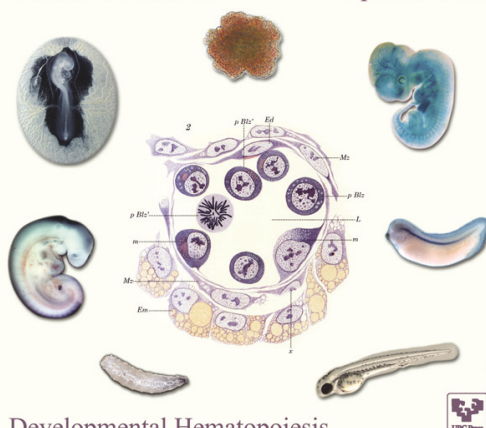

Developmental Hematopoiesis
5 yr ISI Impact Factor $(2011)=2.959$
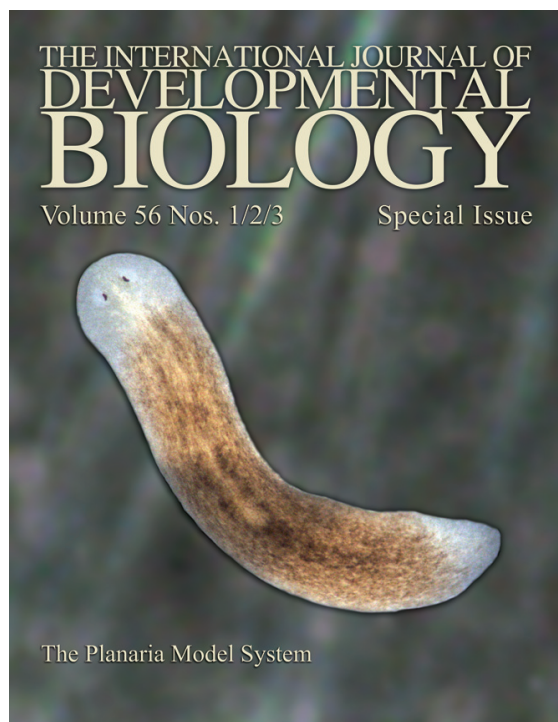
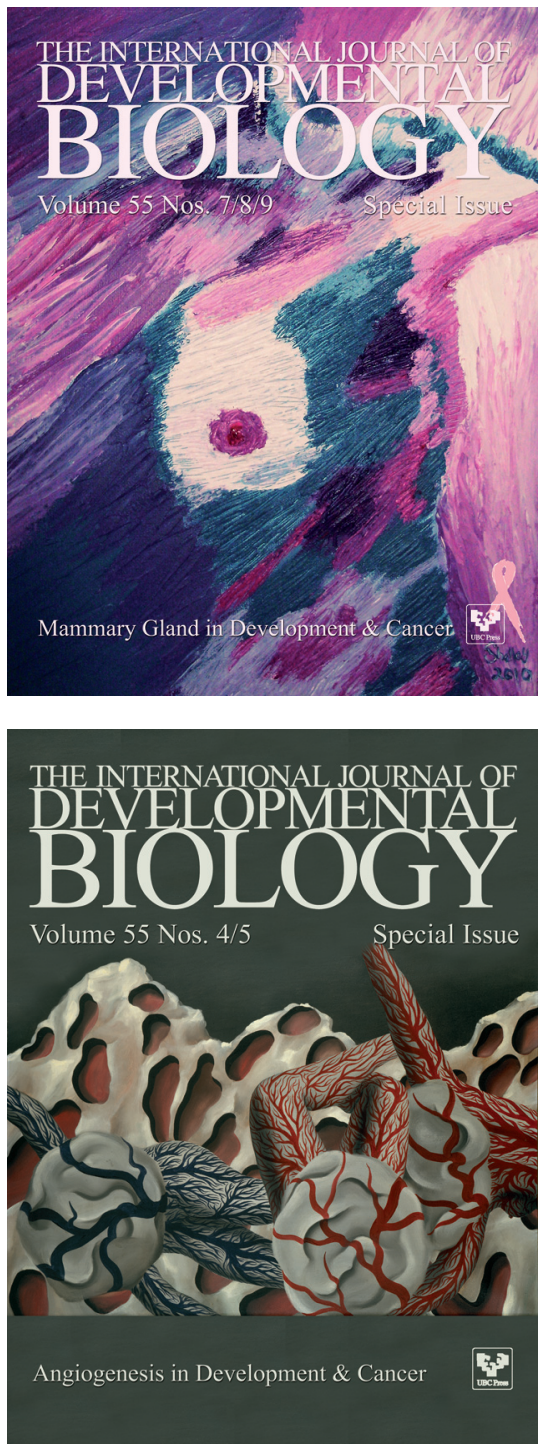